\title{
THERMAL SHOCK IN PERIODIC EDGE-CRACKED PLATE SUPPORTED BY ELASTIC FOUNDATION
}

\author{
AbD El-Fattah A. RizK \\ Department of Science in Engineering,Faculty of Engineering, International Islamic \\ University Malaysia, P.O. Box 10, 50728, Kuala Lumpur, Malaysia \\ e-mail: abdelfattah@iiu.edu.my
}

\begin{abstract}
The study of the transient thermal stress problem for a periodic edge cracks in an elastic plate on an elastic foundations is investigated. This study may also be applied for circumferentially periodic cracked hollow cylinder under transient thermal stresses. Based on previous studies, the cylindrical shell may be modeled by a plate on an elastic foundation. The thermal stresses are generated due to sudden convective cooling on the boundary containing the edge cracks while the other boundary is insulated. The superposition technique is utilized to solve the problem. The perturbation problem is formulated by using the thermal stresses obtained from uncracked problem with opposite sign on the crack surfaces as the only external loading. This leads to hypersingular integral equation with the crack surface displacement as the only unknown function. The main output of this study is the transient stress intensity factors that are evaluated numerically. The parametric studies based on time (Fourier number), crack length, coefficient of heat transfer (Biot number), Periodic crack spacing and the stiffness of elastic foundation are investigated.
\end{abstract}

Keywords: Fracture mechanics, Thermal stresses, Periodic crack, Stress intensity factor.

\section{INTRODUCTION}

The transient thermal stress problem in an elastic plate has been wide investigated since it can be found in many engineering applications. It is well understood that, the thermal shock due to sudden cooling of the surface can result in very high tensile stresses near the surface. These stresses may be occurred catastrophic failure especially in presence of preexisting surface flaws. Many studies have been investigated for a single crack in a semi-infinite and finite plate under thermal shock (see [1-6]). The analyses of thermal stress problems for multiple cracks are also considered in the literature. The problem of a periodic edge cracks with equally spaced in a long strip due to quenching using the boundary element method is investigated by Bahr et al [7]. Wang et al. [8] examined the multiple crack problem in functionally graded materials under thermal loading. The study of periodic cracking in a half plane under convective cooling using the principle of superposition is given by Rizk [9]. Also, the analysis of a periodic array of cracks in an infinite elastic strip under surface cooling and heating are investigated by Rizk [10, 11]. The problem of periodic array of cracks in fully constrained infinite strip under thermal shock is also considered by Rizk [12]. 
In the present work the studying of periodic array of edge crack in an infinite plate on an elastic foundation is investigated. This study will also be useful to investigate the cylindrical shell having circumferentially periodic array of edge cracks under thermal shock. According to previous studies [13], the cylindrical shell can be modeled by a plate on an elastic foundation that may make the shell problem analytically tractable.

In this analysis, it is assumed that the problem is linear. So, the superposition technique is utilized, i.e. the solution of the thermal problem is considered to be the sum of two solutions. The first solution is evaluated for the transient thermal stress problem without cracks. The second solution is obtained for the isothermal periodic crack problem in which the crack surface tractions that are equal and opposite to the thermal stresses obtained from the first solution are the only external loads. In addition, the transient thermal problem is assumed to be quasi-static (i.e. the inertia effects are neglected), and all thermoelastic coupling effects and the temperature dependence of the thermoelastic constants are neglected. Note that, since the cracks are in $x$-direction, they will not disturb the temperature and the stress distributions. By defining the unknown function in terms of crack surface displacement, the perturbation problem will be reduced to hypersingular integral equation. The expansion method is adopted and the principle of finite-part integral developed by Kaya and Erdogan [14, 15] are used for numerical solution.

The important parameter that needs to predict mechanical failure in the subcritical crack growth is the stress intensity. So, the main results of this work are the variation of the stress intensity factor as a function of time (Fourier number), coefficient of heat transfer (Boit number), crack length, periodic crack spacing and the stiffness of elastic foundation.

\section{MATHEMATICAL FORMULATION}

Consider the plane strain problem for an elastic infinite strip of thickness $H$ at initial temperature $T_{o}$ with periodic cracks of length $(b-a)$ spaced out by $2 c$ is elastically supported with coefficient $\eta$ as shown in Fig. 1. The edge crack case will be considered by taking $a=0$. The surface containing the edge cracks $(x=0)$ is suddenly exposed to convective cooling at temperature $T_{a}$ with heat transfer coefficient $h$. The other surface $(x=H)$ that is supported by elastic foundation is assumed to be insulated.

The thermal stresses for the transient thermal stress uncracked problem can be set up by solving first the diffusion equation with proper boundary conditions using Lapalce transform technique to obtain the transient temperature distribution as developed in [5] and it is given by

$$
\frac{\theta\left(x^{*}, \tau\right)}{\theta_{o}}=\frac{T\left(x^{*}, \tau\right)-T_{a}}{T_{o}-T_{a}}=2 \sum_{n=1}^{\infty} \frac{\sin \lambda_{n} \cos \lambda_{n}\left(x^{*}-1\right)}{\lambda_{n}+0.5 \sin 2 \lambda_{n}} e^{-\tau \lambda_{n}^{2}}
$$




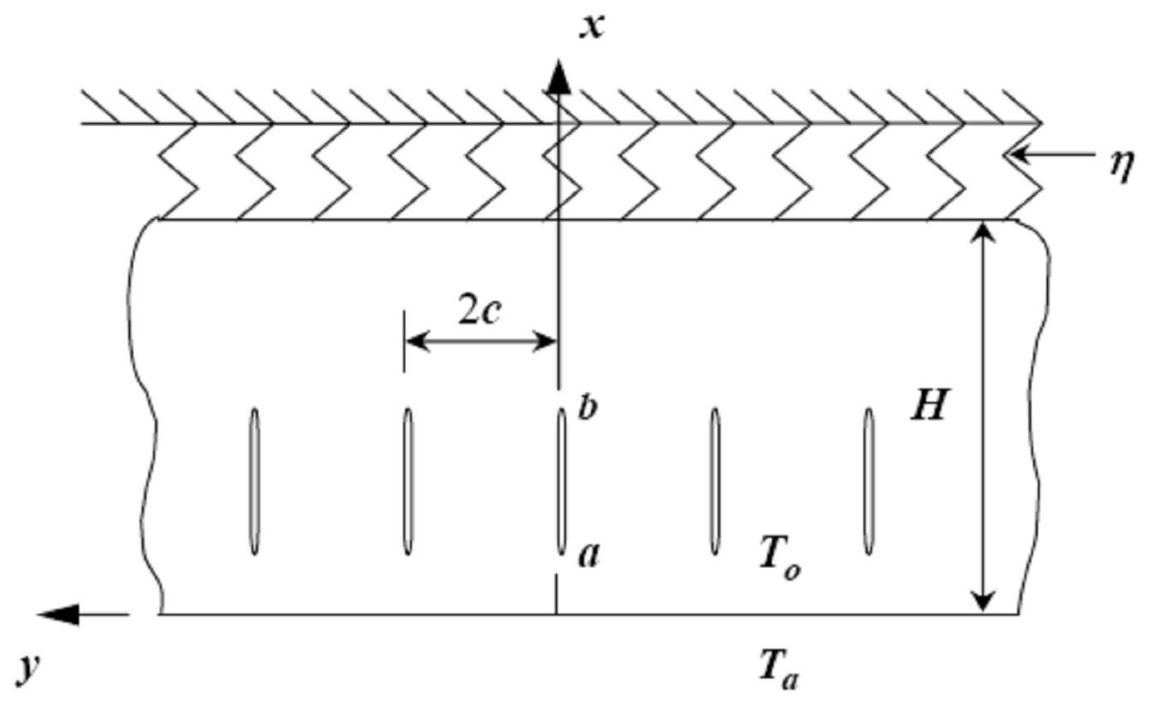

Fig.1: Periodic crack geometry

where, $x^{*}=x / H, \tau=t D / H^{2}$ (Fourier number), $D$ is the thermal diffusivity and $\lambda_{n}$ is the roots of the equation $\lambda_{n} \tan \lambda_{n}=B i$, where $B i$ is the Biot number that is defined by $B i=h H / k^{\prime}$, and $k^{\prime}$ is the material thermal conductivity. The thermal stresses for elastically supported plate can be obtained by observing that the plate will remain flat under self-equilibrating transient thermal stresses, i.e. the plate undergoes uniform strain over the thickness $H$. Following [16] the transient thermal stresses as a result of the transient temperature $\theta(x, t)=T(x, t)-T_{a}$ are given by

$$
\sigma_{y y}^{T}(x, t)=\frac{\alpha E}{(1-v)}\left[\frac{1}{H} \int_{0}^{H} \theta(x, t) d x-\theta(x, t)\right]
$$

where $E$ is the Young's modulus, $v$ is the Poisson's ratio and $\alpha$ is the coefficient of linear thermal expansion. By substituting equation (1) into equation (2), the transient thermal stresses would be in the form

$$
\begin{array}{r}
\frac{\sigma_{y y}^{T}\left(x^{*}, \tau\right)(1-v)}{E \alpha\left(T_{o}-T_{a}\right)}=2 \sum_{n=1}^{\infty}\left[\frac{\sin ^{2} \lambda_{n}}{\lambda_{n}\left(\lambda_{n}+0.5 \sin 2 \lambda_{n}\right)}\right] e^{-\tau \lambda_{n}^{2}} \\
-2 \sum_{n=1}^{\infty}\left[\frac{\sin \lambda_{n} \cos \lambda_{n}\left(x^{*}-1\right)}{\lambda_{n}+0.5 \sin 2 \lambda_{n}}\right] e^{-\tau \lambda_{n}^{2}}
\end{array}
$$

The analysis of the circumferential cracked problem in a cylindrical shell may be approximated by a plate supported by elastic foundation under plane strain conditions. Previous studies showed the validity of this assumption [13] and the stiffness of the elastic foundation is given by 


$$
\eta=\frac{E H}{R_{n}^{2}}
$$

where $E$ is Young's modulus, $H$ is the thickness of the cylinder $\left(R_{o}-R_{i}\right), R_{o}$ is the outer radius, $R_{i}$ is the inner radius, and $R_{n}$ is the mean radius of the cylinder.

The plane strain problem shown in Fig. 1 may be formulated by solving the following governing differential equations

$$
\begin{aligned}
& (\kappa-1) \nabla^{2} u+2\left(\frac{\partial^{2} u}{\partial x^{2}}+\frac{\partial^{2} v}{\partial x \partial y}\right)=0 \\
& (\kappa-1) \nabla^{2} v+2\left(\frac{\partial^{2} u}{\partial x \partial y}+\frac{\partial^{2} v}{\partial y^{2}}\right)=0
\end{aligned}
$$

where $\kappa=(3-4 v)$ for plane strain, $\kappa=(3-v) /(1+v)$ for plane stress, and $u, v$ are the $x$ and $y$ components of the displacement vector. Because of periodicity, the local perturbation problem is considered, i.e. $0<y<c$, and the problem should be solved under the following homogeneous and mixed boundary conditions:

$$
\begin{aligned}
& \sigma_{x y}(x, 0)=0, \sigma_{x y}(x, c)=0, v(x, c)=0, \quad 0<x<H \\
& \sigma_{x x}(0, y)=0, \sigma_{x y}(0, y)=0,0<y<c \\
& \sigma_{x x}(H, y)=\eta u(H, y), \sigma_{x y}(H, y)=0,0<y<c \\
& \left.\begin{array}{lr}
v(x, 0)=0,0<x<a, b<x<H & (a) \\
\sigma_{y y}(x, 0)=-\sigma_{y y}^{T}(x, t), a<x<b & (b)
\end{array}\right\}
\end{aligned}
$$

where $\sigma_{i j}(i, j=x, y)$ are the stresses, $\eta$ is the stiffness of the elastic foundation and $\sigma_{y y}^{T}(x, t)$ is the thermal stress obtained from the uncracked problem. The solution of the mixed boundary value problem described by equations (5)-(10) may be obtained by expressing the displacement components in terms of sums of finite and infinite Fourier transform [17]:

$$
\begin{aligned}
& u(x, y)=\sum_{n=0}^{\infty} f\left(x, \alpha_{n}\right) \cos \alpha_{n} y+\frac{1}{2 \pi} \int_{-\infty}^{\infty} p(y, \beta) e^{i x \beta} d \beta \\
& v(x, y)=\sum_{n=1}^{\infty} g\left(x, \alpha_{n}\right) \sin \alpha_{n} y+\frac{1}{2 \pi} \int_{-\infty}^{\infty} q(y, \beta) e^{i x \beta} d \beta
\end{aligned}
$$


where $\alpha_{n}=\pi n / c$. By substituting equations (11) and (12) into equations (5) and (6), and by solving ordinary differential equations, the displacements $u, v$ may be expressed as

$$
\begin{aligned}
& u(x, y)=\sum_{n=0}^{\infty}\left[\left(C_{1}+C_{2} x\right) e^{x \alpha_{n}}+\left(C_{3}+C_{4} x\right) e^{-x \alpha_{n}}\right] \cos \alpha_{n} y \\
& +\frac{1}{2 \pi} \int_{-\infty}^{\infty} \frac{i|\beta|}{\beta}\left[\left(D_{1}+\frac{\kappa}{|\beta|} D_{2}+D_{2} y\right) e^{y|\beta|}\right. \\
& \left.-\left(D_{3}-\frac{\kappa}{|\beta|} D_{4}+D_{4} y\right) e^{-y|\beta|}\right] e^{i x \beta} d \beta \\
& v(x, y)=\sum_{n=1}^{\infty}\left[\left(-C_{1}-\frac{\kappa}{\alpha_{n}} C_{2}-C_{2} x\right) e^{x \alpha_{n}}\right. \\
& \left.+\left(C_{3}-\frac{\kappa}{\alpha_{n}} C_{4}+C_{4} x\right) e^{-x \alpha_{n}}\right] \sin \alpha_{n} y \\
& +\frac{1}{2 \pi} \int_{-\infty}^{\infty}\left[\left(D_{1}+D_{2} y\right) e^{y|\beta|}+\left(D_{3}+D_{4} y\right) e^{-y|\beta|}\right] e^{i x \beta} d \beta
\end{aligned}
$$

where the unknown functions $D_{i}(i=1,2,3,4)$ and $C_{i}(i=1,2,3,4)$ are functions of the transform variables $\beta$ and $\alpha_{n}$ respectively. Using the stress-strain relations, the straindisplacement relations and equations (13) and (14), the stress components $\sigma_{x x}, \sigma_{y y}, \sigma_{x y}$ may be given by

$$
\begin{aligned}
\frac{1}{2 \mu} \sigma_{x x}(x, y)=\sum_{n=1}^{\infty} & {\left[\left(C_{1} \alpha_{n}+C_{2} x \alpha_{n}+\frac{\kappa-1}{2} C_{2}\right) e^{x \alpha_{n}}\right.} \\
& \left.-\left(C_{3} \alpha_{n}+C_{4} x \alpha_{n}-\frac{\kappa-1}{2} C_{4}\right) e^{-x \alpha_{n}}\right] \cos \alpha_{n} y \\
& +\frac{1}{2 \pi} \int_{-\infty}^{\infty}-\mid \beta\left[\left(D_{1}+D_{2} \frac{\kappa+3}{2|\beta|}+D_{2} y\right) e^{y|\beta|}\right. \\
& \left.-\left(D_{3}-D_{4} \frac{\kappa+3}{2|\beta|}+D_{4} y\right) e^{-y|\beta|}\right] e^{i x \beta} d \beta
\end{aligned}
$$




$$
\begin{aligned}
\frac{1}{2 \mu} \sigma_{y y}(x, y)=\sum_{n=1}^{\infty} & {\left[\left(-C_{1} \alpha_{n}-C_{2} x \alpha_{n}-\frac{\kappa+3}{2} C_{2}\right) e^{x \alpha_{n}}\right.} \\
& \left.+\left(C_{3} \alpha_{n}+C_{4} x \alpha_{n}-\frac{\kappa+3}{2} C_{4}\right) e^{-x \alpha_{n}}\right] \cos \alpha_{n} y \\
& +\frac{1}{2 \pi} \int_{-\infty}^{\infty}|\beta|\left[\left(D_{1}+D_{2} \frac{\kappa-1}{2|\beta|}+D_{2} y\right) e^{y|\beta|}\right. \\
& \left.-\left(D_{3}-D_{4} \frac{\kappa-1}{2|\beta|}+D_{4} y\right) e^{-y|\beta|}\right] e^{i x \beta} d \beta \\
\frac{1}{2 \mu} \sigma_{x y}(x, y)=\sum_{n=1}^{\infty} & {\left[\left(-C_{1} \alpha_{n}-C_{2} x \alpha_{n}-\frac{\kappa+1}{2} C_{2}\right) e^{x \alpha_{n}}\right.} \\
& \left.+\left(-C_{3} \alpha_{n}-C_{4} x \alpha_{n}+\frac{\kappa+1}{2} C_{4}\right) e^{-x \alpha_{n}}\right] \sin \alpha_{n} y \\
& +\frac{1}{2 \pi} \int_{-\infty}^{\infty} i \beta\left[\left(D_{1}+D_{2} \frac{\kappa+1}{2|\beta|}+D_{2} y\right) e^{y|\beta|}\right. \\
& \left.+\left(D_{3}-D_{4} \frac{\kappa+1}{2|\beta|}+D_{4} y\right) e^{-y|\beta|}\right] e^{i x \beta} d \beta
\end{aligned}
$$

where $\mu=E / 2(1+v)$ is the shear modulus. Seven of these unknown functions can be eliminated by the conditions (7-9). By defining the new function $\phi(x)=v(x, 0)$ and using the mixed boundary condition (10), the problem will be reduced to the following singular integral equation for the unknown function $\phi(x)$

$$
\lim _{y \rightarrow 0} \int_{a}^{b}\left[K_{1}(x, y, s)+K_{2}(x, y, s)\right] \varphi(s) d s=-\frac{\pi(\kappa+1)}{4 \mu} \sigma_{y y}^{T}(x, t)
$$

where the kernels $K_{1}, K_{2}$ are given by

$$
\begin{aligned}
& K_{1}(x, y, s)=\int_{0}^{\infty}\left\{\frac{\left[(1-y \beta) e^{-4 c \beta}+(y \beta-2 c \beta-1) e^{-2 c \beta}\right] e^{y \beta}}{\left(1-e^{-2 c \beta}\right)^{2}}\right. \\
& \left.-\frac{\left[(2 c \beta-y \beta-1) e^{-2 c \beta}+(y \beta+1)\right] e^{-y \beta}}{\left(1-e^{-2 c \beta}\right)^{2}}\right\} \beta \cos (s-x) \beta d \beta \\
& K_{2}(x, y, s)=\frac{\pi}{c} \sum_{n=1}^{\infty} \frac{1}{2 M}[I+I I] \cos \alpha_{n} y
\end{aligned}
$$




$$
\begin{aligned}
& I=\left[10 \alpha_{n}-6 \alpha_{n}^{2(}(2 H-x-s)+4 \alpha_{n}^{3}(s-H)(x-H)\right] e^{(s+x-2 H) \alpha_{n}}+ \\
& {\left[6 \alpha_{n}+2 \alpha_{n}^{2}(x-s-10 H)+12 \alpha_{n}^{3} H(H-x+s)+8 \alpha_{n}^{4} s H(x-H)\right] e^{-(s-x+2 H) \alpha_{n}}+} \\
& {\left[-10 \alpha_{n}-6 \alpha_{n}^{2}(x+s)-4 \alpha_{n}^{3} s x\right] e^{(s+x-4 H) \alpha_{n}}+} \\
& {\left[-6 \alpha_{n}+2 \alpha_{n}^{2}(s-x)\right] e^{-(s-x+4 H) \alpha_{n}}} \\
& +\frac{\eta}{2 \mu \alpha_{n}} \frac{\kappa+1}{2}\left\{\left[6 \alpha_{n}-2 \alpha_{n}^{2}(2 H-x-s)\right] e^{(s+x-2 H) \alpha_{n}}+\right. \\
& {\left[10 \alpha_{n}-6 \alpha_{n}^{2}(2 H-x+s)+4 \alpha_{n}^{3} s(2 H-x)\right] e^{-(s-x+2 H) \alpha_{n}}+} \\
& {\left[10 \alpha_{n}+6 \alpha_{n}^{2}(x+s)+4 \alpha_{n}^{3} s x\right] e^{(s+x-4 H) \alpha_{n}}+} \\
& \left.\left[6 \alpha_{n}-2 \alpha_{n}^{2}(s-x)\right] e^{-(s-x+4 H) \alpha_{n}}\right\} \\
& I I=\left[6 \alpha_{n}+2 \alpha_{n}^{2}(s-x-10 H)+12 \alpha_{n}^{3} H(x-s+H)+8 \alpha_{n}^{4} x H(s-H)\right] e^{(s-x-2 H) \alpha_{n}}+ \\
& {\left[10 \alpha_{n}-6 \alpha_{n}^{2}(x+s)+4 \alpha_{n}^{3} s x\right] e^{-(s+x) \alpha_{n}}+} \\
& {\left[-6 \alpha_{n}-2 \alpha_{n}^{2}(s-x)\right] e^{(s-x-4 H) \alpha_{n}}+} \\
& {\left[-12 \alpha_{n}-6 \alpha_{n}^{2}(2 H-x-s)-4 \alpha_{n}^{3}(H-s)(H-x)\right] e^{-(s+x+2 H) \alpha_{n}}} \\
& +\frac{\eta}{2 \mu \alpha_{n}} \frac{\kappa+1}{2}\left\{\left[10 \alpha_{n}-6 \alpha_{n}^{2}(s+x)+4 \alpha_{n}^{3} s x\right] e^{-(s+x) \alpha_{n}}+\right. \\
& {\left[10 \alpha_{n}-6 \alpha_{n}^{2}(2 H+x-s)+4 \alpha_{n}^{3} x(2 H-s)\right] e^{(s-x-2 H) \alpha_{n}}+} \\
& {\left[6 \alpha_{n}+2 \alpha_{n}^{2}(2 H-x-s)\right] e^{-(s+x+2 H) \alpha_{n}}+} \\
& \left.\left[6 \alpha_{n}+2 \alpha_{n}^{2}(s-x)\right] e^{(s-x-4 H) \alpha_{n}}\right\} \\
& M=1-\left(2+4 \alpha_{n}^{2} H^{2}\right) e^{-2 \alpha_{n} H}+e^{-4 \alpha_{n} H}+\frac{\eta}{2 \mu \alpha_{n}} \frac{\kappa+1}{2}\left(1+4 \alpha_{n} H e^{-2 \alpha_{n} H}-e^{-4 \alpha_{n} H}\right)
\end{aligned}
$$

The singular terms must be separated from the kernels $K_{1}$ and $K_{2}$ before solving the integral equation (18). By examining the asymptotic behavior of $K_{1}$ for $\beta \rightarrow \infty$, $(s-x) \rightarrow 0$ and taking the limit $y \rightarrow 0$, it is found that

$$
\lim _{y \rightarrow 0} K_{1}(x, y, s)=\frac{1}{(s-x)^{2}}+k_{1}^{f}(x, s)
$$

where $k_{1}^{f}(x, s)$ is bounded term and it is given by

$$
k_{1}^{f}(x, s)=\int_{0}^{\infty}\left[\frac{2 e^{-4 c \beta}-2(2 c \beta+1) e^{-2 c \beta}}{\left(1-e^{-2 c \beta}\right)^{2}}\right] \beta \cos (s-x) \beta d \beta
$$


The kernel $K_{2}$ is bounded for embedded $\operatorname{crack}(a>0, b<H)$, and as the crack approaches the boundary $(a=0)$ some terms will be unbounded and the asymptotic behavior of $K_{2}$ for $n \rightarrow \infty$ and $(s+x) \rightarrow 0$ would be

$$
\lim _{y \rightarrow 0} K_{2}(x, y, s)=k_{2}^{f}(x, s)+k_{2 a}^{\infty}(x, s)
$$

where $k_{2}^{f}(x, s)$ is bounded term and $k_{2 a}^{\infty}$ is unbounded term that is given by

$$
k_{2 a}^{\infty}(x, s)=\lim _{y \rightarrow 0} \frac{\pi}{c} \sum_{n=1}^{\infty}\left[5 \alpha_{n}-3 \alpha_{n}^{2}(s+x)+2 \alpha_{n}^{3} s x\right] e^{-(s+x) \alpha_{n}} \cos \alpha_{n} y
$$

After summing the series and taking the limit, the generalized singular terms may also be separated i.e.

$$
k_{2 a}^{\infty}(x, s)=k_{2 a}^{f}(x, s)+k_{2 a}^{s}(x, s)
$$

where

$$
k_{2 a}^{s}(x, s)=\frac{-1}{(s+x)^{2}}+\frac{12 x}{(s+x)^{3}}-\frac{12 x^{2}}{(s+x)^{4}}
$$

Then the singular integral equation (18) may be expressed as

$$
\int_{a}^{b} \frac{\varphi(s)}{(s-x)^{2}} d s+\int_{a}^{b} k(x, s) \varphi(s) d s=-\frac{\pi(\kappa+1)}{4 \mu} \sigma_{y y}^{T}(x, t)
$$

where

$$
k(x, s)=k_{1}^{f}(x, s)+k_{2}^{f}(x, s)+k_{2 a}^{f}(x, s)+k_{2 a}^{s}(x, s)
$$

The solution of the integral equation would be based on the behavior of the unknown function $\phi(s)$ that can be obtained by following Muskhelishvili technique [18] and the concept of finite part-integral described by Kaya and Erdogan $[14,15]$. Let $\phi(s)$ will be in the form

$$
\varphi(s)=f(s)(s-a)^{\gamma_{1}}(b-s)^{\gamma_{2}}
$$

where the unknown function $f(s)$ is bounded with $f(a) \neq 0, f(b) \neq 0$, and $\gamma_{1}, \gamma_{2}$ would depend on the location of the crack tip. It can be shown that for an impeded crack $\gamma_{1}=\gamma_{2}=1 / 2(a>0, b<H)$, and for an edge crack $(a=0, b<H) \gamma_{1}=0, \gamma_{2}=1 / 2$. By defining the stress intensity factor at the crack tip $b$ as 


$$
K(b)=\lim _{x \rightarrow b} \sqrt{2(x-b)} \sigma_{y y}(x, 0)=\frac{4 \mu}{\kappa+1} \lim _{x \rightarrow b} \frac{v(x, 0)}{\sqrt{2(b-x)}}
$$

and since $v(x, 0)=f(x) \sqrt{b-x}$ for an edge crack, the stress intensity factor at the edge $b$ becomes

$$
K(b)=\frac{4 \mu}{\kappa+1} \frac{f(b)}{\sqrt{2}}
$$

\section{NUMERICAL SOLUTION}

To evaluate the stress intensity factor given by equation (28), it is required to solve the singular integral equation (25) for the unknown function $f(s)$. First it is normalized by the following variables

$$
\begin{array}{ll}
x=\frac{b-a}{2} r+\frac{b+a}{2}, & s=\frac{b-a}{2} \rho+\frac{b+a}{2} \\
\phi(s)=\left(\frac{b-a}{2}\right) \psi(\rho), & h(r, \rho)=\left(\frac{b-a}{2}\right)^{2} k(x, s)
\end{array}
$$

giving

$$
\int_{-1}^{1} \frac{\psi(\rho)}{(\rho-r)^{2}} d \rho+\int_{-1}^{1} h(r, \rho) \psi(\rho) d \rho=-\frac{\pi(\kappa+1)}{4 \mu} \sigma_{y y}^{T}(r, t),-1<r<1
$$

Also from equations (26) and (29), $\psi(\rho)$ may be written as

$$
\psi(\rho)=F(\rho)(1+\rho)^{\gamma_{1}}(1-\rho)^{\gamma_{2}}
$$

where $F(\rho)$ is the new unknown function that may be approximated by truncated series as

$$
F(\rho)=\sum_{n=0}^{N} a_{n} \rho^{n}
$$

where $a_{n}$ are $(N+1)$ unknown coefficients to be determined. After substituting equations (32) and (33) into equation (31), we end up with $(N+1)$ linear equations that are solved at certain collocation points which are selected to be to the zeros of Chebychev polynomial of $\operatorname{order}(N+1)$, i.e.

$$
r_{j}=\cos \frac{\pi(2 j-1)}{2(N+1)} \quad, j=1,2, \ldots, N+1
$$


Following the technique developed by Kaya and Erdogan [14,15], the unknown coefficients $a_{n}$ can be determined and then the stress intensity factors can be evaluated.

\section{RESULTS}

Figure 2 shows sample results of the normalized transient thermal stresses versus normalized distance $x / H$ calculated from equation (3) for two values of Biot number $B i=\infty, 20$ and different values of normalized time (Fourier number) $\tau=0.001,0.01$, $0.1,0.5,1.0$. From the stress profiles it can be observed that, for $B i=\infty$ which corresponds to the unit step function temperature change on the boundary leads to highest transient thermal stresses compared to the values obtained for $B i=20$. At any instant of time the thermal stresses is tensile in the region near the cooled surface and compressive in region near the insulated surface which satisfy zero resultant force at any cross section of the plate. Also the effect of the time (Fourier number) on thermal stresses can be seen in the figure. The gradient of the thermal stresses is decreasing as the time increases and reaching zero value for large time.
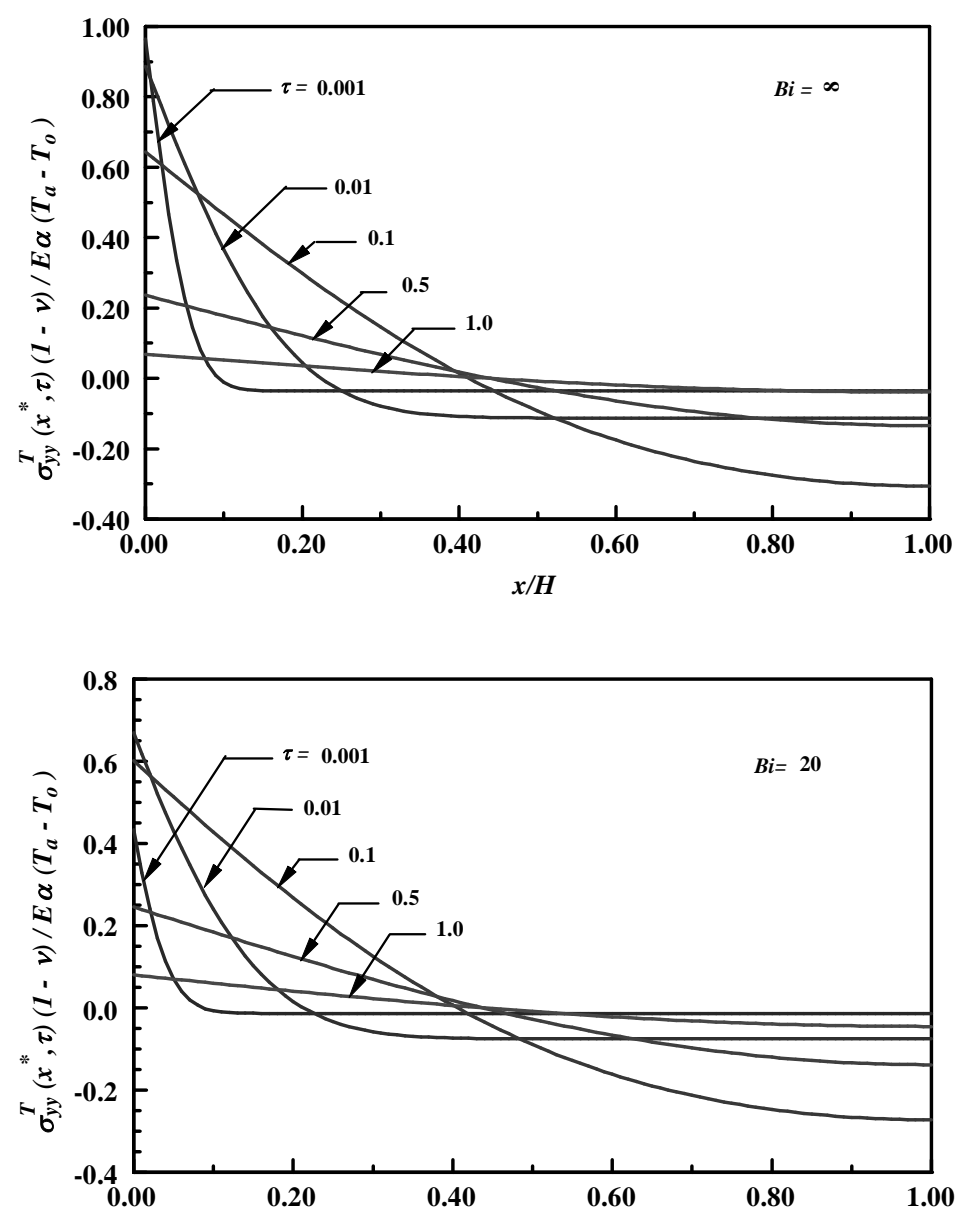

Fig. 2: Transient thermal stressexs ${ }^{x / 4}$ for $B i=\infty$ and $B i=20$ 
The influence of the normalized stiffness of the elastic foundation $\eta H / E$ on the normalized stress intensity factors defined by $K(b)(1-v) / E \alpha\left(T_{o}-T_{a}\right) \sqrt{b}$ is given in Fig. 3 for different values of $\eta H / E=0.00238,0.01108,0.08163,1.0$, $\infty$ which are corresponding to $R_{i} / H=20,9,3,0.5$ respectively, except for $\eta H / E=\infty$ which is related to fully constrained boundary as given by Rizk [12]. . The results are obtained for normalized crack length $b / H=0.3$, Biot number $B i=\infty$, which is the most severe case, and three values of normalized periodic crack spacing $b / 2 c=0.05,0.1,0.3$. Infact, the values of $R_{i} / H=3$ and 0.5 represent thick hollow cylinders which can not be modeled by a plate on an elastic foundation. The figure shows great effect of the normalized stiffness $\eta H / E$ on the normalized stress intensity factors especially for small normalized periodic crack spacing $(b / 2 c=0.05)$. As the normalized stiffness increases the normalized stress intensity factors decrease and the smallest values would be for the case of fully constrained boundary $\eta H / E=\infty$. The effect of the normalized crack spacing $(b / 2 c)$ on the normalized stress intensity factors is also exposed in the figure. If the numbers of the cracks increase per unit length $(b / 2 c$ increases) the normalized stress intensity factors are decreasing consequently and the effect of the normalized stiffness becomes less. For $b / 2 c=0.1$ the values of the normalized stress intensity factors for $\eta H / E=0.00238,0.01108\left(R_{i} / H=20,9\right)$ are almost the same. For $b / 2 c=0.3$ the values of the normalized stress intensity factors are approximately equal for all given values of $\eta H / E=0.00238,0.01108,0.08163,1.0, \infty$ and they are represented in the figure by one curve which corresponds to the fully constrained boundary problem. So the elastic foundation will perform as a fully constrained boundary when the cracks become closer to each other.

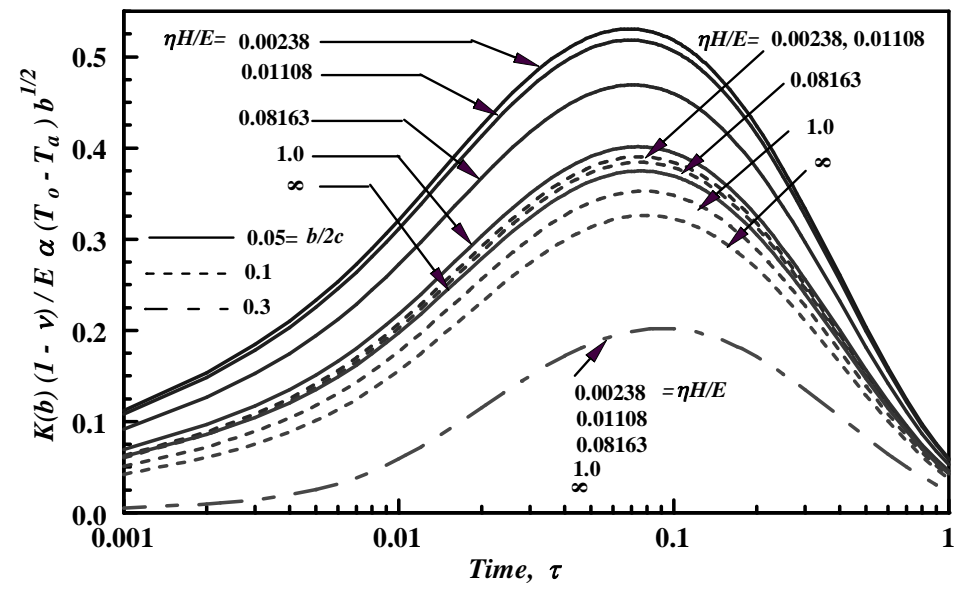

Fig. 3: Stress intensity factors for different values of $\eta H / E, b / 2 c=0.05,0.1,0.3$,

$$
b / H=0.3, B i=\infty
$$

The variation of the normalized stress intensity factors versus time is represented in Fig. 4 for varying values of normalized periodic crack spacing $b / 2 c=0.0,0.1,0.2$, $0.3,0.5$. The results are shown for $b / H=0.4, B i=\infty$ and two values of normalized 
stiffness $\eta H / E=0.01108, \infty$. It can be seen that the normalized stress intensity factors decrease as the normalized periodic spacing increases ( $c$ decreases) and the most dangerous case would occur for $b / 2 c=0.0$ which is related to single crack problem. For large normalized periodic crack spacing $(b / 2 c=0.5)$ the variation of the normalized stress intensity factors for $\eta H / E=0.01108, \infty$ are almost identical and they would approach the fully constrained boundary case $(\eta H / E=\infty)$. Also for large $b / 2 c$ the normalized stress intensity factors start to be negative while they are always positive for single crack $(b / 2 c=0.0)$. The negative values of the normalized stress intensity factors are meaningless because crack closure occur, or it is meaningful only when these results are used in a superposition and the stress intensity factors are positive.

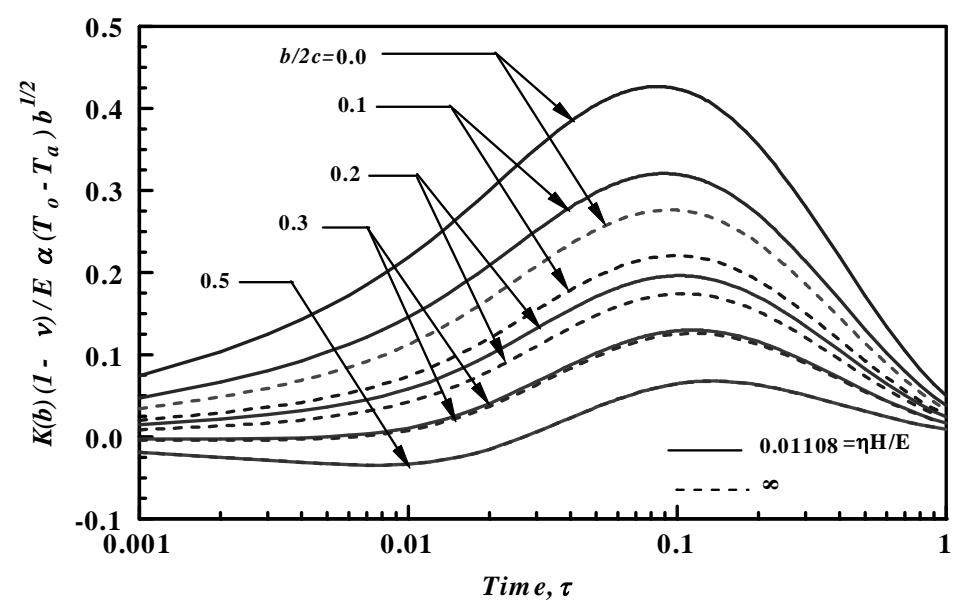

Fig. 4: Stress Intensity factors for different values of $b / 2 c, \eta H / E=0.01108, \infty$,

$$
b / H=0.4, B i=\infty
$$

Figure 5 displays the variation of the normalized stress intensity factors versus normalized time for varying normalized crack length $b / H=0.05,0.1,0.20 .3,0.4$, 0.5 . The calculations are based on $b / 2 c=0.1, B i=\infty$ and two values of normalized stiffness $\eta H / E=0.01108, \infty$. As expected, the largest normalized stress intensity factors would occur for small crack which lies completely in the tensile region and as the normalized crack length increases the normalized stress intensity factors decrease due to the increasing effect of the compressive stresses in the interior of the plate. Noted that, for small crack $(b / H=0.05,0.1)$, The normalized stiffness has weak effect on the normalized stress intensity factors while it has great effect for large crack $(b / H=0.4,0.5)$. 


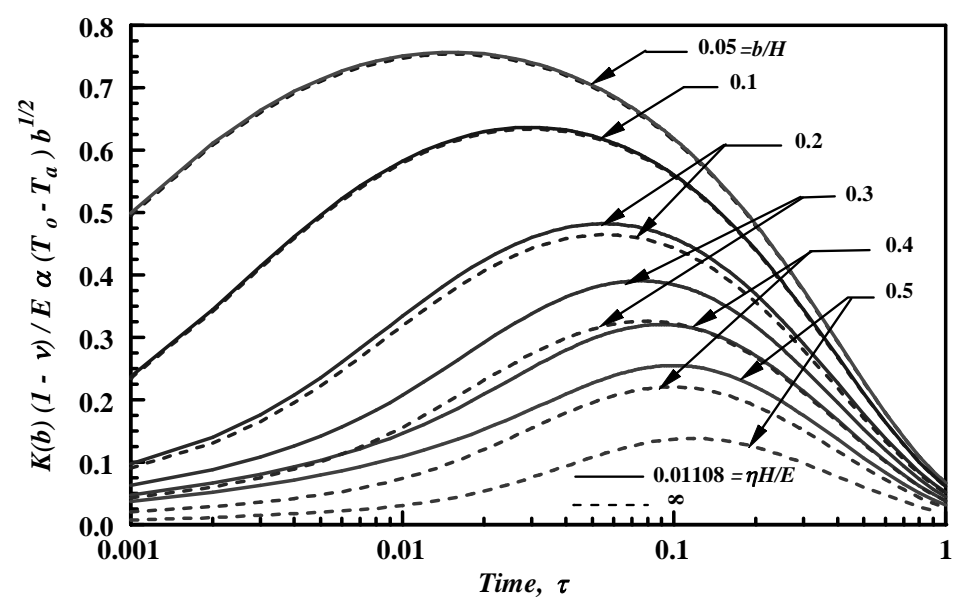

Fig. 5: Stress intensity factors for different values of $b / H, \eta H / E=0.01108, \infty$,

$$
b / 2 c=0.1, B i=\infty
$$

Finally, Fig. 6 shows the effect of the Biot number on the normalized stress intensity factors for different values of $B i=1,5,10,20, \infty$ and two values of normalized crack length $\quad b / H=0.1,0.5$. The results are obtained for $b / 2 c=0.1$ and $\eta H / E=0.01108$. It is clear that the normalized stress intensity factors are reducing by decreasing the Biot number due to the decreasing in the thermal stresses and the most severe case will be for unit step function temperature change on the boundary $(\beta=\infty)$. The effect of the normalized crack length on the normalized stress intensity factors is also shown in the figure by reducing it as the $b / H$ increases.

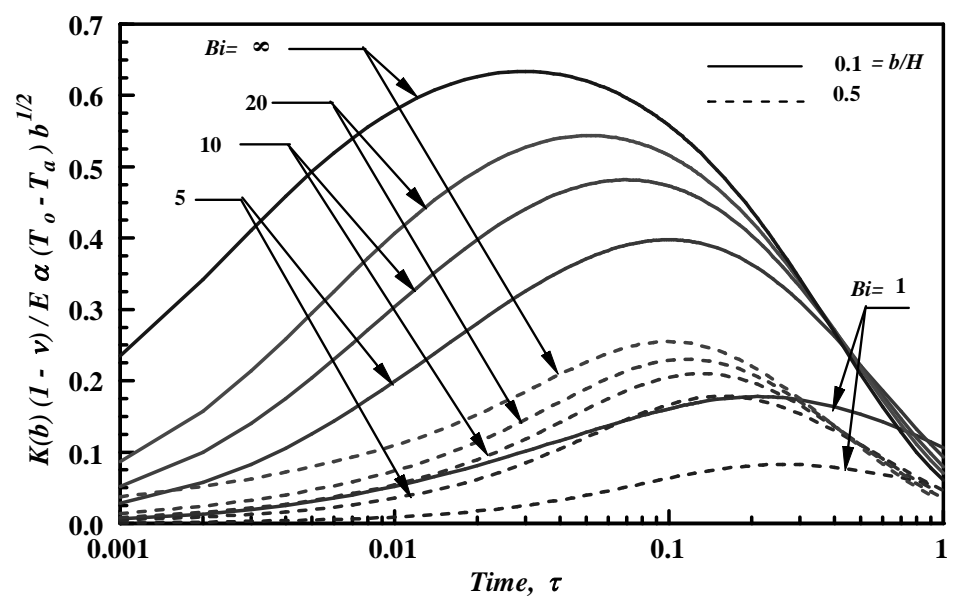

Fig. 6: Stress intensity factors for different values of $B i, b / H=0.1,0.5$,

$$
\eta H / E=0.01108, b / 2 c=0.1 \text {. }
$$




\section{CONCLUSION}

In this paper, the effect of the stiffness of elastic foundation $(\eta H / E)$, the periodic crack spacing $(b / 2 c)$, the heat transfer coefficient (Biot number, $B i)$, the crack length $(b / H)$ and the time (Fourier number, $\tau=t D / H^{2}$ ) on the stress intensity factors are investigated. The stiffness of the elastic foundation has quit effect on the stress intensity factors especially for large crack and large periodic crack spacing ( $b / 2 c$ small) by reducing the normalized stress intensity factors as the normalized stiffness increases. For small crack the elastic foundation has very small effect on the stress intensity factors. By increasing the crack length the normalized stress intensity factors decrease accordingly. The periodic crack spacing has a great effect on the stress intensity factors. As $b / 2 c$ increases ( $c$ decreases), the crack interaction will reduce the the normalized stress intensity factors and the effect of the stiffness of the elastic foundation will approach the fully constrained boundary case. In addition the increasing $b / H$ and $b / 2 c$ the normalized stress intensity factors started to be negative leading to the crack surfaces to be in contact along certain contact length from the edge crack $b$. Also it is shown that the normalized stress intensity factors would be reduced with decreasing the Biot number.

\section{REFERENCES}

[1] H.F. Nied, "Thermal shock in an edge-cracked plate," Journal of Thermal stresses, Vol.1, pp. 217-227, 1983.

[2] H.-A. Bahr, H. Balke, M. Kuna, and H. Liesk, "Fracture analysis of a single edge cracked strip under thermal shock," Theoretical Applied Fracture Mechanics, Vol. 8, pp. 33-39, 1987.

[3] A.A. Rizk and S.F. Radwan, "Transient thermal stress problem for a cracked semi-Infinite medium, "Journal of Thermal Stresses, vol. 15, pp. 451-468, 1992.

[4] X. Fan and S. Yu, "Thermal shock in a surface-cracked plate", Engineering Fracture Mechanics, vol. 41, 1992.

[5] A.A. Rizk, "Edge-cracked plate with one free and one constrained boundary subjected to sudden convective cooling," Journal of Thermal Stresses, Vol. 1, pp. 455-471, 1994.

[6] A.A Rizk, "Fracture of shells and plates on an elastic foundation under convective cooling", Alexandria Engineering Journal, vol. 33, no. 3, pp. 121127, 1994.

[7] H.A. Bahr, H.J. Weiss, H.G. Maschke, and F. Meissner, "Multiple crack propagation in a strip caused by thermal shock," Theoretical and Applied Fracture Mechanics, vol. 10, pp. 219-226, 1988.

[8] B.L., Wang, J.C. Han, S.Y Du, "Thermoelastic fracture mechanics for nonhomogenous material subjected to unsteady thermal load," ASME Journal of Applied Mechanics, vol. 67, pp. 87-95, 2000.

[9] A.A. Rizk, "Transient stress intensity factors for periodic array of cracks in a half-plane due to convective cooling, " Journal of Thermal Stresses, vol. 26, pp. 443-456, 2003.

[10] A.A. Rizk, "Peiodic array of cracks in a strip subjected to surface heating," International Journal of Solids and Structures, Vol. 41, pp. 4685-4696, 2004.

[11] A.A. Rizk, "Convective thermal shock of an infinite plate with periodic edge cracks", Journal of thermal stresses, vol.28, pp. 103-119, 2005. 
[12] A.A. Rizk, "Periodic edge cracks in fully constrained infinite Elastic strip under Thermal Shock", 8th International Conference on Production Engineering, Design, and Control (PEDAC'2004), Alexandria University, Alexandria, Egypt, 27-29 Dec. 2004.

[13] A.P. Boresi and R.J. Schmidt, "Advanced Mechanics of Materials", 6th Edition, John Wiley, 2003.

[14] A.C. Kaya and F. Erdogan, "On the solution of integral equations with strongly singular kernels," Quarterly of Applied Mathematics, XLV, pp. 105-122, 1987.

[15] A.C. Kaya, and F. Erdogan, "On the solution of integral equations with generalized Cauchy kernel," Quarterly of Applied Mathematics, XLV, pp.455-469, 1987.

[16] B.A. Boley and J.H Weiner, "Theory of Thermal Stresses", John Wiley, 1960.

[17] GW. Schulze and F. Erdogan, "Periodic cracking of elastic coatings," International Journal of Solids and Structures, Vol. 35, pp. 3615-3634, 1998.

[18] N.I. Muskhelishvili, "Singular Integral Equations," Noordhoff, Groningen, the Netherlands, 1953. 